\title{
Confounders in Adenoma Detection at Initial Screening Colonoscopy: A Factor in the Assessment of Racial Disparities as a Risk for Colon Cancer
}

\author{
Yakira David1,2* (1), Lorenzo Ottaviano3 ${ }^{3}$, Jihye Park ${ }^{4}$, Sadat Iqbal', Michelle Likhtshteyn', \\ Samir Kumar1, Helen Lyo', Ayanna E. Lewis ${ }^{3}$, Brandon E. Lung3, Jesse T. Frye3 ${ }^{3}$, Li Huang3, \\ Ellen $\mathrm{Li}^{3}$, Jie Yang ${ }^{5}$, Laura Martello ${ }^{1}$, Shivakumar Vignesh ${ }^{1}$, Joshua D. Miller ${ }^{3}$, Michele Follen, \\ Evan B. Grossman ${ }^{1,7}$
}

${ }^{1}$ Department of Medicine, SUNY Downstate Medical Center, Brooklyn, NY, USA

${ }^{2}$ Department of Gastroenterology, Icahn School of Medicine at Mount Sinai, New York City, NY, USA

${ }^{3}$ Department of Medicine, Stony Brook University, Stony Brook, NY, USA

${ }^{4}$ Department of Applied Mathematics and Statistics, Stony Brook University, Stony Brook, NY, USA

${ }^{5}$ Department of Family, Population and Preventive Medicine, Stony Brook University, Stony Brook, NY, USA

${ }^{6}$ Department of Obstetrics and Gynecology, NYC HNC/Kings County, Brooklyn, NY, USA

${ }^{7}$ Department of Medicine, Division of Gastroenterology, NYC Health and Hospitals/Kings County, Brooklyn, NY, USA

Email: *yakiradavid@gmail.com

How to cite this paper: David, Y., Ottaviano, L., Park, J., Iqbal, S., Likhtshteyn, M., Kumar, S., Lyo, H., Lewism A.E., Lung, B.E., Frye, J.T., Huang, L., Li, E., Yang, J., Martello, L., Vignesh, S., Miller, J.D., Follen, M. and Grossman, E.B. (2019) Confounders in Adenoma Detection at Initial Screening Colonoscopy: A Factor in the Assessment of Racial Disparities as a Risk for Colon Cancer. Journal of Cancer Therapy, 10, 269-289.

https://doi.org/10.4236/jct.2019.104022

Received: March 5, 2019

Accepted: April 6, 2019

Published: April 9, 2019

Copyright (๑) 2019 by author(s) and Scientific Research Publishing Inc. This work is licensed under the Creative Commons Attribution International License (CC BY 4.0).

http://creativecommons.org/licenses/by/4.0/

\begin{abstract}
Background and Aims: The incidence and mortality of colorectal cancer is persistently highest in Black/African-Americans in the United States. While access to care, barriers to screening, and poverty might explain these findings, there in increased interest in examining biological factors that impact the colonic environment. Our group is examining biologic factors that contribute to disparities in development of adenomas prospectively. In preparation for this and to characterize a potential patient population, we conducted a retrospective review of initial screening colonoscopies in a cohort of patients. Methods: A retrospective review was performed on initial average risk screening colonoscopies on patients (age 45 - 75 years) during 2012 at three institutions. Descriptive statistics and multivariable logistic regression models were used to examine the relationship between potential risk factors and the detection of adenomas. Results: Of the 2225 initial screening colonoscopies 1495 (67.2\%) were performed on Black/African-Americans and 566 (25.4\%) on Caucasians. Multivariable logistic regression revealed that older age, male sex, current smoking and teaching gastroenterologists were associated with higher detection of adenomas and these were less prevalent among Black/AfricanAmericas except for age. Neither race, ethnicity, BMI, diabetes mellitus, HIV
\end{abstract}


nor insurance was associated with adenoma detection. Conclusion: In this sample, there was no association between race and adenoma detection. While this may be due to a lower prevalence of risk factors for adenomas in this sample, our findings were confounded by a lower detection rate by consultant gastroenterologists at one institution. The study allowed us to rectify the problem and characterize patients for future trials.

\section{Keywords}

Colorectal, Cancer, Screening, Adenoma, Quality

\section{Introduction}

Colorectal Cancer (CRC) is the fourth most common cancer in the United States (US) and second most lethal [1]. Most colon cancers develop via a multistep process involving a series of somatic genetic mutations and histopathologic changes that accumulate over time that is estimated to take approximately 10 15 years [2] [3] [4]. Consequently, screening with removal of adenomas and early detection of colorectal cancers has contributed to substantial decreases in the incidence and mortality of colorectal cancer in the United States over the past 10 years [5] [6] [7] [8].

Multiple studies have reported increased risk of colon cancer regardless of racial group to be associated with age, male sex, family history in a first degree relative, smoking, as well as diabetes mellitus and BMI which can be a reflection of diet and activity level [9] [10]. At the molecular level, inflammatory processes are associated with colon tumorigenesis [11]. We hypothesize that these factors also contribute to a higher incidence of precancerous colon polyps and sought to characterize the impact of these factors in our patients.

The incidence and mortality of colon cancer remains significantly higher in Blacks/African-Americans than all other races and ethnicities in this US [1] [12]. This persistent disparity is likely multifactorial in etiology. The incidence and risk of colon cancer is thought to be low in Africa. This may be a reflection of lower rates of detection due to lower screening rates. However investigators have suggested that the higher incidence in US Blacks/African-Americans may be due to biological risk factors as a result of gene-environment interactions [13] [14] [15] [16]. Additionally, disparities in socioeconomic status could contribute to unequal access to colon cancer screening here and abroad [17] [18]. We hypothesize that African-Americans have more risk factors for colorectal cancer and are also more likely to have a higher prevalence of adenomas.

To test these hypotheses, we conducted a retrospective chart review of initial screening colonoscopies performed at three collaborating institutions: SUNY Downstate Medical Center (DMC), New York City Health and Hospitals/Kings County (Kings County), and Stony Brook University Hospital (SBUMC). DMC and SBUMC are funded by New York State while Kings County is supported by 
the New York Health and Hospital Corporation. DMC and Kings County are in central Brooklyn and SBUMC is in Long Island, New York. All three institutions educate residents and fellows and all three employ consultant gastroenterologists from time to time.

\section{Methods}

\section{Collection of clinical data from initial screening colonoscopies performed in 2012}

This study was approved by the Institutional Review Boards for all three institutions (IRB \# 802718 for DMC and Kings County, approved 07/21/2017; IRB \# 966231 for SBUMC, approved 03/21/2017). Patients who underwent screening colonoscopies between January 1 and December 31, 2012, were identified using the endoscopy reporting software at each of the three institutions.

Patients age $<45$ y or $>75 \mathrm{y}$, a history of previous colonoscopy, a history of inflammatory bowel diseases, known hereditary colorectal syndromes, detection of microscopic or macroscopic blood in stool and other alarm symptoms, detection of colonic masses or polyps on previous studies, were excluded from this analysis. We excluded colonoscopies that were incomplete (did not reach the cecum) and those associated with poor bowel preparation.

The clinical metadata was collected using the same data vocabulary at the three institutions and included: 1) age (y) at time of initial screening colonoscopy; 2) sex (male/female); 3) race (Black/Caucasian/Other); 4) ethnicity (Hispanic/non-Hispanic); 5) BMI $\left.\left(\mathrm{kg} / \mathrm{m}^{2}\right) ; 6\right)$ diabetes mellitus (diagnosed, not diagnosed); 7) smoking (current/ not current); 8) HIV-1 (diagnosed/not diagnosed); 9) gastroenterologist (teaching versus consultant); and 10) insurance status (Commercial, Medicare, Medicaid, Self-pay). Patients who had both Commercial and Medicare insurance were classified in the Commercial category. Patients who had both Medicare and Medicaid insurance were classified in the Medicare category. Family history of colon cancer or a polyp in a first degree relative, was not included in the analysis since that data was unevenly collected.

2225 patients with colonoscopy screening were used in this analysis: DMC (N $=444,20 \%)$, Kings County $(\mathrm{N}=1134,51 \%)$, SBUMC $(\mathrm{N}=647,29 \%)$. The outcomes of interest were: adenomas, advanced adenomas, and right colon adenomas.

\section{Statistical analysis}

Descriptive statistics and multivariable logistic regression models were used to examine the relationship between potential risk factors and the detection of three different types of adenomas (all adenomas, advanced adenomas, and right colon adenomas). Due to the high correlation between "race" and "institution", three sets of multivariable logistic regression models were fitted for each clinical outcome: 1) both "race" and "institution" were used in the model; 2) only "race" was used in the model; and 3) only "institution" was used in the model. Since, in general, significant risk factors from 3 multivariable regression models were consistent, and based on c-index values, results from the models that contained both "race" and "institution" were reported here. 
In each multivariable regression analysis, an $\mathrm{OR}>1$ indicates that one category has more risk of having adenoma detection than the reference category, and $\mathrm{OR}<1$ indicates that one category has less risk of having adenoma detection than the reference category. Generalized linear mixed models considering patients from the same institution as clusters were also considered. However, since there is no strong evidence that patients with same institution were highly correlated, results from logistic regression models were only reported here. Statistical analysis was performed using SAS 9.4 and significance level was set at 0.05 (SAS Institute, Inc., Cary, NC).

\section{Results}

Table 1 displays the descriptive table for clinical outcomes and patients' demographics stratified by institution. Based on this table, marginally, all variables were significantly associated with the institutions. For example, $29.73 \%$ of patients from DMC had adenoma, while $17.11 \%$ of patients from Kings County had adenoma, and $25.97 \%$ of patients from SBUMC had adenoma (P-value ${ }^{1}<$ $0.0001)$

Table 2 describes patients' demographics by the clinical outcomes of interest: adenoma, advanced adenoma, and right colonic adenoma. Based on this table, marginally, age, gender, insurance, institution, race, type of attending, tobacco use, diabetes, and HIV-negative status were significantly associated with having an adenoma. For example, $28.54 \%$ of patients having adenoma had diabetes, while $23.74 \%$ of patients who do not have adenoma had diabetes ( $\mathrm{P}$-value $=$ 0.0294). For advanced adenoma, institution, type of attending, fellow, and tobacco use were marginally associated with having this outcome. For right colon adenoma, age, gender, insurance, institution, and type of attending were marginally associated with having this outcome.

Table 3 shows the results of estimated Odds Ratios (ORs) and 95\% confidence intervals of potential risk factors for adenomas based on multivariable logistic regression model. After adjusting for other factors, age, gender, tobacco use, and HIV-negative status remain significantly associated with having an adenoma (P-values < 0.05). For example, female patients were significantly less likely to have adenoma than male patients $(\mathrm{OR}=0.626,95 \% \mathrm{CI}$ : $0.5-0.78$, P-value $<$ $0.0001)$.

Table 4 shows the results of estimated ORs and 95\% confidence intervals of explanatory variables for having advanced adenoma based on multivariable logistic regression model. Due to the limited event size $(\mathrm{N}=98)$, forward selection was further performed in the multivariable regression model. After adjusting for other factors, insurance, fellow (fellows accompanied academic gastroenterologists and not consultants), and tobacco use were significantly associated with having an advanced adenoma. For example, patients who currently smoke were significantly more likely to have advanced adenoma than patients who do not smoke $\left(\mathrm{OR}=2.362,95 \%\right.$ CI: $\left.1.37-4.06, \mathrm{P}_{\text {-value }}^{2}=0.0019\right)$. 
Table 1. Descriptive table for clinical outcomes and patients' demographics by 3 institutions.

\begin{tabular}{|c|c|c|c|c|c|c|c|}
\hline Variable & Missing & level & total & $\begin{array}{c}\text { DMC } \\
(\mathrm{N}=444)\end{array}$ & $\begin{array}{l}\text { Kings county } \\
(\mathrm{N}=1134)\end{array}$ & $\begin{array}{l}\text { SBUMC } \\
(\mathrm{N}=647)\end{array}$ & P-value ${ }^{1}$ \\
\hline \multirow{2}{*}{ Adenoma } & \multirow{2}{*}{0} & Yes & $494(22.20 \%)$ & $132(29.73 \%)$ & $194(17.11 \%)$ & $168(25.97 \%)$ & \multirow{2}{*}{$<0.0001$} \\
\hline & & No & $1731(77.80 \%)$ & $312(70.27 \%)$ & $940(82.89 \%)$ & $479(74.03 \%)$ & \\
\hline \multirow{2}{*}{ Advanced adenoma } & \multirow{2}{*}{0} & Yes & $98(4.40 \%)$ & $15(3.38 \%)$ & $42(3.70 \%)$ & $41(6.34 \%)$ & \multirow{2}{*}{0.0168} \\
\hline & & No & $2127(95.60 \%)$ & $429(96.62 \%)$ & $1092(96.30 \%)$ & $606(93.66 \%)$ & \\
\hline \multirow{2}{*}{ Right colon adenoma } & \multirow{2}{*}{0} & Yes & $316(14.20 \%)$ & $77(17.34 \%)$ & $142(12.52 \%)$ & 97 (14.99\%) & \multirow{2}{*}{0.0378} \\
\hline & & No & $1909(85.80 \%)$ & $367(82.66 \%)$ & $992(87.48 \%)$ & $550(85.01 \%)$ & \\
\hline \multirow[t]{2}{*}{ Age (continuous) } & 0 & 444 vs 1134 vs 647 & $57.00 \pm 10.73$ & $58.00 \pm 12.00$ & $57.63 \pm 10.73$ & $55.00 \pm 10.00$ & \multirow[t]{2}{*}{$<0.0001$} \\
\hline & & $45-54$ & 879 (39.51\%) & $144(32.43 \%)$ & $438(38.62 \%)$ & $297(45.90 \%)$ & \\
\hline \multirow[t]{2}{*}{ Age (categorical) } & 0 & $55-64$ & $919(41.30 \%)$ & $187(42.12 \%)$ & $490(43.21 \%)$ & $242(37.40 \%)$ & \multirow[t]{2}{*}{$<0.0001$} \\
\hline & & $\geq 65$ & 427 (19.19\%) & $113(25.45 \%)$ & $206(18.17 \%)$ & $108(16.69 \%)$ & \\
\hline \multirow[t]{2}{*}{ BMI (continuous) } & 34 & 413 vs 1131 vs 647 & $28.27 \pm 7.29$ & $28.30 \pm 7.80$ & $28.60 \pm 7.30$ & $27.48 \pm 6.44$ & \multirow[t]{2}{*}{0.0002} \\
\hline & & $\begin{array}{c}\text { Obese } \\
(\mathrm{BMI} \geq 30)\end{array}$ & $829(37.82 \%)$ & $166(40.10 \%)$ & $460(40.67 \%)$ & $203(31.38 \%)$ & \\
\hline \multirow[t]{2}{*}{ BMI (categorical) } & \multirow[t]{2}{*}{33} & $\begin{array}{c}\text { Overweight } \\
(\mathrm{BMI} \geq 25,<30)\end{array}$ & $844(38.50 \%)$ & $147(35.51 \%)$ & $430(38.02 \%)$ & $267(41.27 \%)$ & \multirow[t]{2}{*}{0.0015} \\
\hline & & $\begin{array}{c}\text { Underweight + Healthy } \\
\quad(\mathrm{BMI}<25)\end{array}$ & $519(23.68 \%)$ & $101(24.40 \%)$ & $241(21.31 \%)$ & $177(27.36 \%)$ & \\
\hline \multirow{3}{*}{ Gender } & \multirow{3}{*}{0} & Female & $1437(64.58 \%)$ & $276(62.16 \%)$ & $772(68.08 \%)$ & $389(60.12 \%)$ & \multirow{2}{*}{0.0016} \\
\hline & & Male & $788(35.42 \%)$ & $168(37.84 \%)$ & $362(31.92 \%)$ & $258(39.88 \%)$ & \\
\hline & & Private & $759(34.11 \%)$ & $129(29.05 \%)$ & $195(17.20 \%)$ & $435(67.23 \%)$ & \multirow{4}{*}{$<0.0001$} \\
\hline \multirow{5}{*}{ Insurance } & \multirow{3}{*}{0} & Medicare & $236(10.61 \%)$ & $95(21.40 \%)$ & $57(5.03 \%)$ & $84(12.98 \%)$ & \\
\hline & & Medicaid & $695(31.24 \%)$ & $187(42.12 \%)$ & $404(35.63 \%)$ & $104(16.07 \%)$ & \\
\hline & & Self-Pay & $535(24.04 \%)$ & $33(7.43 \%)$ & $478(42.15 \%)$ & $24(3.71 \%)$ & \\
\hline & \multirow{7}{*}{0} & African-American & $1117(50.20 \%)$ & $175(39.41 \%)$ & $900(79.37 \%)$ & $42(6.49 \%)$ & \multirow{7}{*}{$<0.0001$} \\
\hline & & Afro-Caribbean & 378 (16.99\%) & $216(48.65 \%)$ & $160(14.11 \%)$ & $2(0.31 \%)$ & \\
\hline \multirow[t]{5}{*}{ Race } & & $\begin{array}{l}\text { American Indian or } \\
\text { Alaskan Native }\end{array}$ & $2(0.09 \%)$ & $0(0.00 \%)$ & $1(0.09 \%)$ & $1(0.15 \%)$ & \\
\hline & & Asian & $43(1.93 \%)$ & $6(1.35 \%)$ & $9(0.79 \%)$ & $28(4.33 \%)$ & \\
\hline & & Other & $119(5.35 \%)$ & $13(2.93 \%)$ & $59(5.20 \%)$ & $47(7.26 \%)$ & \\
\hline & & White & $566(25.44 \%)$ & $34(7.66 \%)$ & $5(0.44 \%)$ & $527(81.45 \%)$ & \\
\hline & & White & $566(25.44 \%)$ & $34(7.66 \%)$ & $5(0.44 \%)$ & $527(81.45 \%)$ & \\
\hline \multirow[t]{2}{*}{ Race combined } & 0 & Black & $1495(67.19 \%)$ & $391(88.06 \%)$ & $1060(93.47 \%)$ & $44(6.80 \%)$ & $<0.0001$ \\
\hline & & Other & $164(7.37 \%)$ & $19(4.28 \%)$ & $69(6.08 \%)$ & $76(11.75 \%)$ & \\
\hline Ethoicity & 0 & Hispanic & $134(6.02 \%)$ & $33(7.43 \%)$ & $46(4.06 \%)$ & $55(8.50 \%)$ & 00002 \\
\hline & & Non-Hispanic & $2091(93.98 \%)$ & $411(92.57 \%)$ & 1088 (95.94\%) & $592(91.50 \%)$ & \\
\hline & & Consultant & $1058(47.55 \%)$ & $25(5.63 \%)$ & $976(86.07 \%)$ & $57(8.81 \%)$ & \\
\hline Type of Attending & 0 & Teaching & $1167(52.45 \%)$ & $419(94.37 \%)$ & $158(13.93 \%)$ & $590(91.19 \%)$ & $<0.0001$ \\
\hline
\end{tabular}




\section{Continued}

\begin{tabular}{|c|c|c|c|c|c|c|c|}
\hline \multirow[b]{2}{*}{ Fellow } & \multirow[b]{2}{*}{0} & Yes & 247 (11.10\%) & $6(1.35 \%)$ & $132(11.64 \%)$ & $109(16.85 \%)$ & \multirow[b]{2}{*}{$<0.0001$} \\
\hline & & No & $1978(88.90 \%)$ & $438(98.65 \%)$ & $1002(88.36 \%)$ & $538(83.15 \%)$ & \\
\hline \multirow{2}{*}{ Tobacco use } & \multirow{2}{*}{68} & Current & $202(9.36 \%)$ & $53(14.10 \%)$ & $66(5.82 \%)$ & $83(12.83 \%)$ & \multirow{2}{*}{$<0.0001$} \\
\hline & & Not current & 1955 (90.64\%) & $323(85.90 \%)$ & $1068(94.18 \%)$ & $564(87.17 \%)$ & \\
\hline \multirow{2}{*}{ Diabetes } & \multirow{2}{*}{0} & Yes & $552(24.81 \%)$ & $135(30.41 \%)$ & 339 (29.89\%) & $78(12.06 \%)$ & \multirow{2}{*}{$<0.0001$} \\
\hline & & No & $1673(75.19 \%)$ & 309 (69.59\%) & $795(70.11 \%)$ & $569(87.94 \%)$ & \\
\hline \multirow{2}{*}{ HIV Status } & \multirow{2}{*}{1} & Yes & $85(3.82 \%)$ & $38(8.56 \%)$ & $42(3.70 \%)$ & $5(0.77 \%)$ & \multirow{2}{*}{$<0.0001$} \\
\hline & & No & $2139(96.18 \%)$ & $406(91.44 \%)$ & $1092(96.30 \%)$ & 641 (99.23\%) & \\
\hline \multirow{3}{*}{ Insulin Use } & \multirow{3}{*}{7} & Yes & $165(7.44 \%)$ & $37(8.45 \%)$ & $114(10.05 \%)$ & $14(2.17 \%)$ & \multirow{3}{*}{$<0.0001$} \\
\hline & & No & $380(17.13 \%)$ & $92(21.00 \%)$ & 225 (19.84\%) & $63(9.75 \%)$ & \\
\hline & & No diabetes & $1673(75.43 \%)$ & $309(70.55 \%)$ & 795 (70.11\%) & $569(88.08 \%)$ & \\
\hline \multirow{3}{*}{ Metformin Use } & \multirow{3}{*}{8} & Yes & 365 (16.46\%) & $60(13.70 \%)$ & $253(22.33 \%)$ & $52(8.05 \%)$ & \multirow{3}{*}{$<0.0001$} \\
\hline & & No & $179(8.07 \%)$ & $69(15.75 \%)$ & 85 (7.50\%) & $25(3.87 \%)$ & \\
\hline & & No diabetes & $1673(75.46 \%)$ & 309 (70.55\%) & 795 (70.17\%) & $569(88.08 \%)$ & \\
\hline
\end{tabular}

${ }^{1}$ For categorical variables, P-value was based on Chi-squared test with exact P-value from Monte Carlo simulation. For continuous variables, P-value was based on Wilcoxon rank sum test and median with IQR were reported. *Since 1 patient from DMC institution had BMI as " $>30$ ”, this patient was treated as having missing value when using BMI as continuous values.

Table 2. Descriptive table for patients' demographics by 3 clinical outcomes.

\begin{tabular}{|c|c|c|c|c|c|c|}
\hline & & & Adenoma & & & \\
\hline Variable & Missing & Level & Total & $\begin{array}{c}\text { Yes } \\
(\mathrm{N}=494,22.2 \%)\end{array}$ & $\begin{array}{c}\text { No } \\
(\mathrm{N}=1731,77.8 \%)\end{array}$ & P-value ${ }^{1}$ \\
\hline \multirow[t]{2}{*}{ Age (continuous) } & 0 & 494 vs 1731 & $57.00 \pm 10.73$ & $58.76 \pm 11.39$ & $56.85 \pm 10.84$ & $<0.0001$ \\
\hline & & $45-54$ & $879(39.51 \%)$ & $159(32.19 \%)$ & $720(41.59 \%)$ & \\
\hline \multirow[t]{2}{*}{ Age (categorical) } & 0 & $55-64$ & 919 (41.30\%) & $216(43.72 \%)$ & $703(40.61 \%)$ & 0.0002 \\
\hline & & $\geq 65$ & 427 (19.19\%) & $119(24.09 \%)$ & $308(17.79 \%)$ & \\
\hline \multirow[t]{2}{*}{ BMI (continuous) } & 34 & 481 vs 1710 & $28.27 \pm 7.29$ & $28.00 \pm 6.50$ & $28.30 \pm 7.35$ & 0.1718 \\
\hline & & $\begin{array}{c}\text { Obese } \\
(\mathrm{BMI} \geq 30)\end{array}$ & $829(37.82 \%)$ & $168(34.85 \%)$ & $661(38.65 \%)$ & \\
\hline \multirow[t]{2}{*}{ BMI (categorical) } & 33 & $\begin{array}{c}\text { Overweight } \\
(\mathrm{BMI} \geq 25,<30)\end{array}$ & $844(38.50 \%)$ & 199 (41.29\%) & $645(37.72 \%)$ & 0.2609 \\
\hline & & $\begin{array}{l}\text { Underweight }+ \text { Healthy } \\
\qquad(\mathrm{BMI}<25)\end{array}$ & $519(23.68 \%)$ & $115(23.86 \%)$ & $404(23.63 \%)$ & \\
\hline \multirow[b]{2}{*}{ Gender } & \multirow[b]{2}{*}{0} & Female & $1437(64.58 \%)$ & $275(55.67 \%)$ & $1162(67.13 \%)$ & \multirow[b]{2}{*}{$<0.0001$} \\
\hline & & Male & $788(35.42 \%)$ & $219(44.33 \%)$ & $569(32.87 \%)$ & \\
\hline \multirow[b]{3}{*}{ Insurance } & & Private & $759(34.11 \%)$ & $179(36.23 \%)$ & $580(33.51 \%)$ & \multirow[b]{3}{*}{$<0.0001$} \\
\hline & & Medicare & $236(10.61 \%)$ & 79 (15.99\%) & 157 (9.07\%) & \\
\hline & 0 & Medicaid & $695(31.24 \%)$ & $142(28.74 \%)$ & 553 (31.95\%) & \\
\hline
\end{tabular}




\section{Continued}

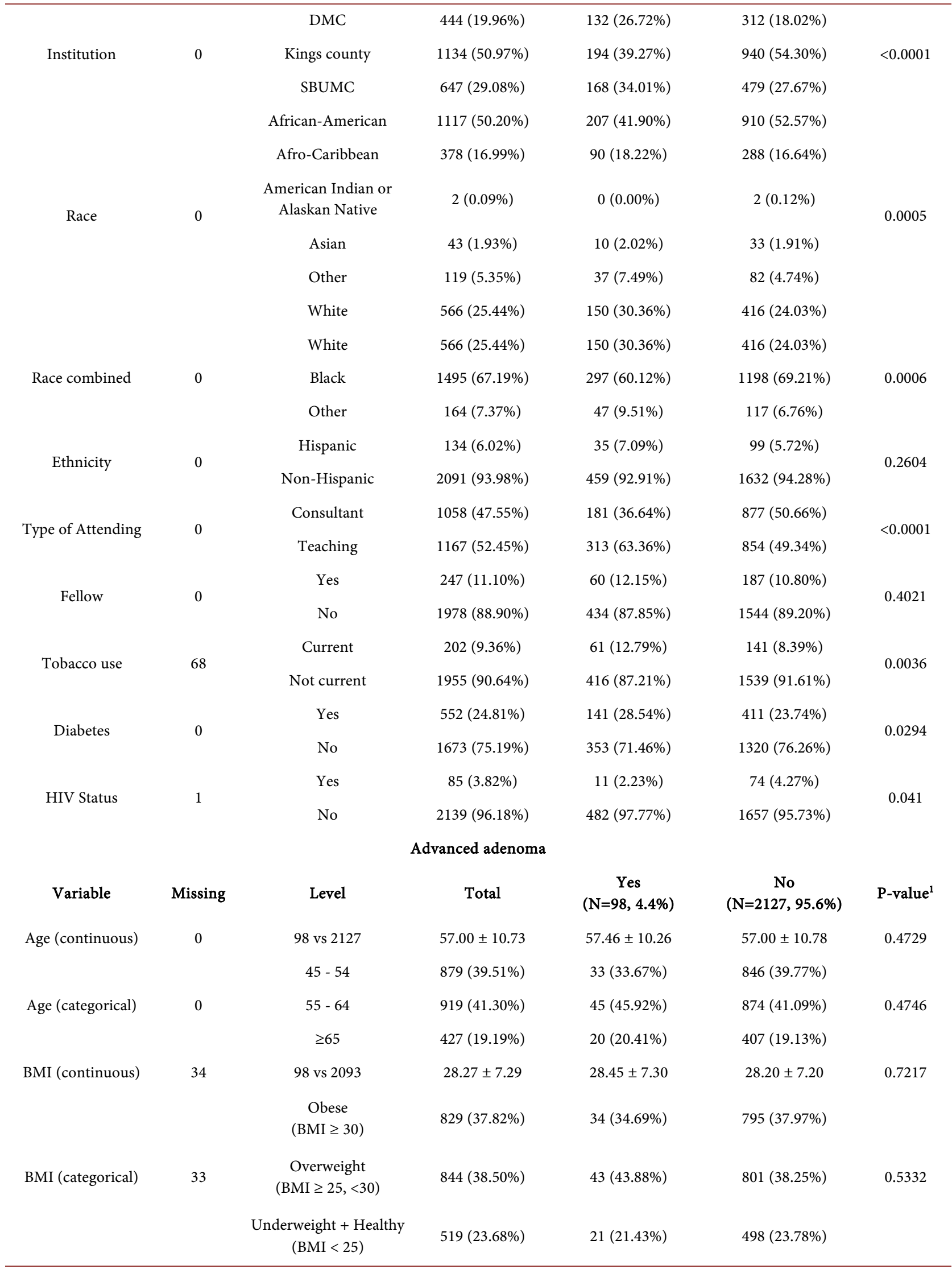




\section{Continued}

\begin{tabular}{|c|c|c|c|c|c|c|}
\hline \multirow{2}{*}{ Gender } & \multirow{2}{*}{0} & Female & $1437(64.58 \%)$ & $55(56.12 \%)$ & $1382(64.97 \%)$ & \multirow{2}{*}{0.0732} \\
\hline & & Male & $788(35.42 \%)$ & $43(43.88 \%)$ & 745 (35.03\%) & \\
\hline \multirow{4}{*}{ Insurance } & \multirow{4}{*}{0} & Private & $759(34.11 \%)$ & $31(31.63 \%)$ & $728(34.23 \%)$ & \multirow{4}{*}{0.0691} \\
\hline & & Medicare & $236(10.61 \%)$ & $18(18.37 \%)$ & $218(10.25 \%)$ & \\
\hline & & Medicaid & 695 (31.24\%) & $25(25.51 \%)$ & $670(31.50 \%)$ & \\
\hline & & Self-Pay & $535(24.04 \%)$ & $24(24.49 \%)$ & $511(24.02 \%)$ & \\
\hline \multirow{3}{*}{ Institution } & \multirow{3}{*}{0} & $\mathrm{DMC}$ & $444(19.96 \%)$ & $15(15.31 \%)$ & $429(20.17 \%)$ & \multirow{3}{*}{0.0168} \\
\hline & & Kings county & $1134(50.97 \%)$ & $42(42.86 \%)$ & $1092(51.34 \%)$ & \\
\hline & & SBUMC & 647 (29.08\%) & $41(41.84 \%)$ & $606(28.49 \%)$ & \\
\hline \multirow{6}{*}{ Race } & \multirow{7}{*}{0} & African-American & $1117(50.20 \%)$ & $41(41.84 \%)$ & $1076(50.59 \%)$ & \multirow{7}{*}{0.35} \\
\hline & & Afro-Caribbean & 378 (16.99\%) & $17(17.35 \%)$ & $361(16.97 \%)$ & \\
\hline & & $\begin{array}{l}\text { American Indian or } \\
\text { Alaskan Native }\end{array}$ & $2(0.09 \%)$ & $0(0.00 \%)$ & $2(0.09 \%)$ & \\
\hline & & Asian & $43(1.93 \%)$ & $1(1.02 \%)$ & $42(1.97 \%)$ & \\
\hline & & Other & $119(5.35 \%)$ & $8(8.16 \%)$ & $111(5.22 \%)$ & \\
\hline & & White & $566(25.44 \%)$ & $31(31.63 \%)$ & $535(25.15 \%)$ & \\
\hline \multirow{3}{*}{ Race combined } & & White & $566(25.44 \%)$ & $31(31.63 \%)$ & $535(25.15 \%)$ & \\
\hline & \multirow[t]{2}{*}{0} & Black & 1495 (67.19\%) & $58(59.18 \%)$ & $1437(67.56 \%)$ & \multirow[t]{2}{*}{0.2252} \\
\hline & & Other & $164(7.37 \%)$ & $9(9.18 \%)$ & $155(7.29 \%)$ & \\
\hline \multirow{2}{*}{ Ethnicity } & \multirow{2}{*}{0} & Hispanic & $134(6.02 \%)$ & $5(5.10 \%)$ & $129(6.06 \%)$ & \multirow{2}{*}{0.6953} \\
\hline & & Non-Hispanic & 2091 (93.98\%) & $93(94.90 \%)$ & 1998 (93.94\%) & \\
\hline \multirow{2}{*}{ Type of Attending } & \multirow{2}{*}{0} & Consultant & $1058(47.55 \%)$ & $37(37.76 \%)$ & $1021(48.00 \%)$ & \multirow{2}{*}{0.047} \\
\hline & & Teaching & $1167(52.45 \%)$ & $61(62.24 \%)$ & $1106(52.00 \%)$ & \\
\hline \multirow{2}{*}{ Fellow } & \multirow{2}{*}{0} & Yes & $247(11.10 \%)$ & $19(19.39 \%)$ & $228(10.72 \%)$ & \multirow{2}{*}{0.0076} \\
\hline & & No & $1978(88.90 \%)$ & $79(80.61 \%)$ & $1899(89.28 \%)$ & \\
\hline \multirow{2}{*}{ Tobacco use } & \multirow{2}{*}{68} & Current & $202(9.36 \%)$ & $18(18.37 \%)$ & $184(8.94 \%)$ & \multirow{2}{*}{0.0034} \\
\hline & & Not current & 1955 (90.64\%) & $80(81.63 \%)$ & 1875 (91.06\%) & \\
\hline \multirow{2}{*}{ Diabetes } & & Yes & $552(24.81 \%)$ & $29(29.59 \%)$ & $523(24.59 \%)$ & \\
\hline & 0 & No & $1673(75.19 \%)$ & $69(70.41 \%)$ & $1604(75.41 \%)$ & 0.2022 \\
\hline & & Yes & $85(3.82 \%)$ & $1(1.02 \%)$ & $84(3.95 \%)$ & \\
\hline Hiv status & 1 & No & $2139(96.18 \%)$ & $97(98.98 \%)$ & $2042(96.05 \%)$ & 0.1001 \\
\hline & & & Right colon adenon & & & \\
\hline Variable & Missing & Level & Total & $\begin{array}{c}\text { Yes } \\
(\mathrm{N}=316,14.2 \%)\end{array}$ & $\begin{array}{c}\text { No } \\
(\mathrm{N}=1909,85.8 \%)\end{array}$ & P-value ${ }^{1}$ \\
\hline Age (continuous) & 0 & 316 vs 1909 & $57.00 \pm 10.73$ & $58.96 \pm 10.49$ & $57.00 \pm 10.98$ & $<0.0001$ \\
\hline & & $45-54$ & $879(39.51 \%)$ & $98(31.01 \%)$ & $781(40.91 \%)$ & \\
\hline Age (categorical) & 0 & $55-64$ & $919(41.30 \%)$ & $142(44.94 \%)$ & 777 (40.70\%) & 0.002 \\
\hline & & $\geq 65$ & 427 (19.19\%) & 76 (24.05\%) & 351 (18.39\%) & \\
\hline
\end{tabular}




\section{Continued}

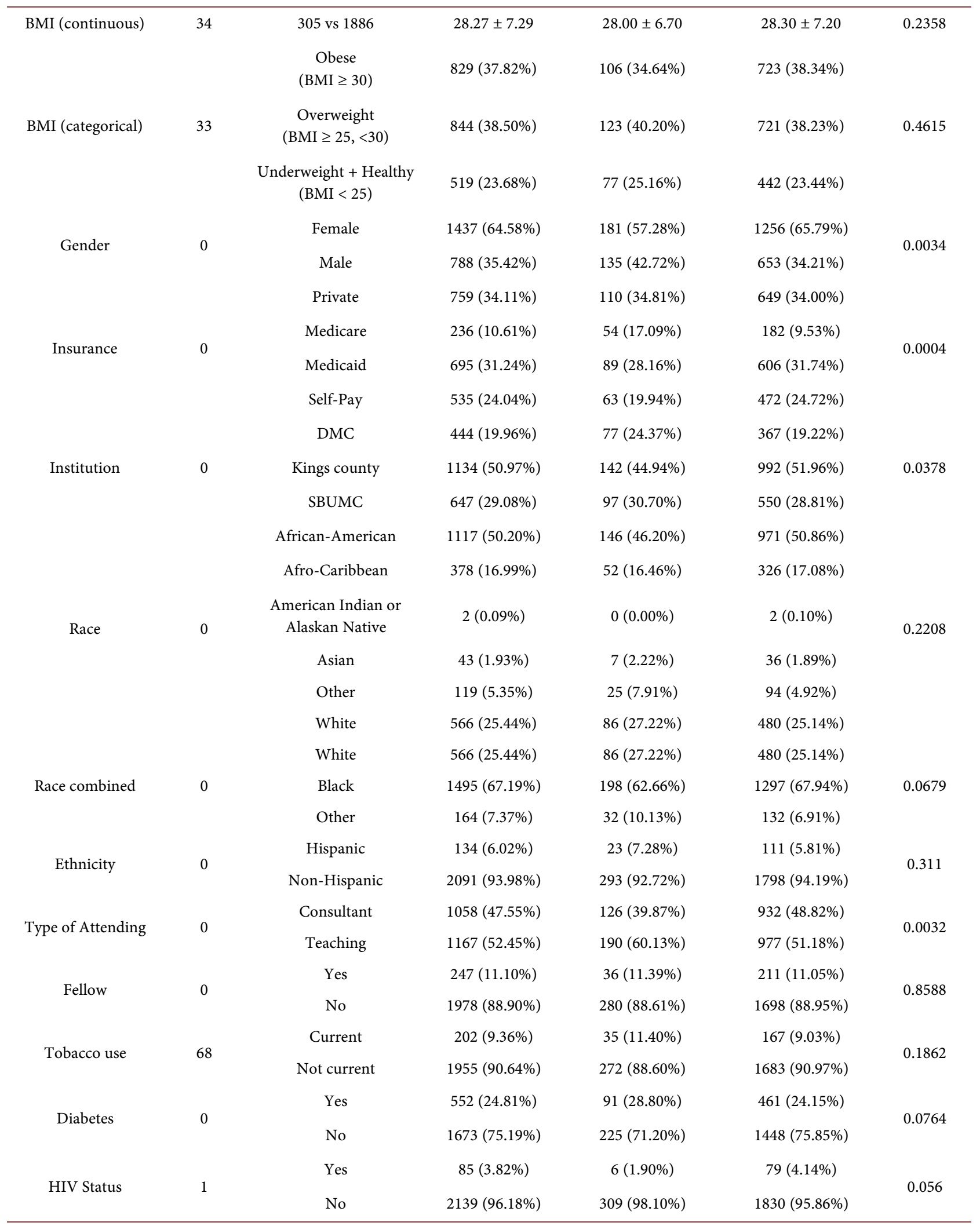

${ }^{1}$ For categorical variables, $\mathrm{P}$-value was based on Chi-squared test with exact P-value from Monte Carlo simulation. For continuous variables, P-value was based on Wilcoxon rank sum test and median with IQR were reported. *Since 1 patient from DMC institution had BMI as ">30", this patient was treated as having missing value when using BMI as continuous values. 
Table 3. Estimated odds ratio and their $95 \%$ confidence intervals of all potential risk factors for Adenoma based on multivariable logistic regression model (c-index: 0.647).

\begin{tabular}{|c|c|c|c|}
\hline Variable & Levels & Odds Ratio (95\% CI) & P-value ${ }^{2}$ \\
\hline Age & Every 1 year increase in Age & $1.027(1.01-1.04)$ & 0.0009 \\
\hline BMI & Every 1 unit increase in BMI & $0.987(0.97-1.01)$ & 0.1658 \\
\hline Gender & Female vs Male & $0.626(0.5-0.78)$ & $<0.0001$ \\
\hline \multirow{6}{*}{ Insurance } & Private vs Medicare & $0.767(0.53-1.1)$ & \multirow{6}{*}{0.404} \\
\hline & Private vs Medicaid & $1.053(0.79-1.4)$ & \\
\hline & Private vs Self-Pay & $1.003(0.71-1.41)$ & \\
\hline & Medicare vs Medicaid & $1.373(0.94-1.99)$ & \\
\hline & Medicare vs Self-Pay & $1.308(0.86-2)$ & \\
\hline & Medicaid vs Self-Pay & $0.953(0.69-1.31)$ & \\
\hline \multirow{3}{*}{ Institution } & DMC vs Kings county & $1.515(0.94-2.43)$ & \multirow{3}{*}{0.0694} \\
\hline & DMC vs SBUMC & $1.605(0.99-2.59)$ & \\
\hline & Kings county vs SBUMC & $1.059(0.59-1.89)$ & \\
\hline \multirow{3}{*}{ Race combined } & White vs Black & $1.426(0.89-2.29)$ & \multirow{3}{*}{0.1116} \\
\hline & White vs Other & $0.849(0.53-1.37)$ & \\
\hline & Black vs Other & $0.596(0.36-0.98)$ & \\
\hline Ethnicity & Hispanic vs Non-Hispanic & $0.986(0.6-1.62)$ & 0.957 \\
\hline Type of attending & Consultant vs Teaching & $0.741(0.49-1.12)$ & 0.1543 \\
\hline Fellow & Yes vs No & $1.176(0.8-1.74)$ & 0.4155 \\
\hline Tobacco use & Current vs Not current & $1.499(1.07-2.11)$ & 0.0195 \\
\hline Diabetes & Yes vs No & $1.265(0.98-1.63)$ & 0.066 \\
\hline HIV status & Yes vs No & $0.473(0.24-0.95)$ & 0.0348 \\
\hline
\end{tabular}

${ }^{2} \mathrm{P}$-value was based on type3 analysis from multivariable logistic regression model.

Table 4. Estimated odds ratio and their $95 \%$ confidence intervals of all potential risk factors for advanced adenoma based on multivariable logistic regression model (c-index: $0.612)$.

\begin{tabular}{cccc}
\hline Variable & Levels & Odds Ratio $(95 \%$ CI $)$ & P-value $^{2}$ \\
\hline Private vs Medicare & $0.448(0.24-0.82)$ & \\
Private vs Medicaid & $1.137(0.66-1.95)$ & \\
Private vs Self-Pay & $0.875(0.51-1.51)$ & 0.0245 \\
Insurance & Medicare vs Medicaid & $2.54(1.35-4.78)$ & \\
& Medicare vs Self-Pay & $1.953(1.03-3.7)$ & \\
& Medicaid vs Self-Pay & $0.769(0.43-1.37)$ & \\
Fellow & Yes vs No & $1.912(1.13-3.24)$ & 0.0158 \\
Tobacco use & Current vs Not current & $2.362(1.37-4.06)$ & 0.0019 \\
\hline
\end{tabular}

${ }^{2} \mathrm{P}$-value was based on type3 analysis from multivariable logistic regression model. 
Table 5 shows the results of estimated ORs and 95\% confidence intervals of explanatory variables for having right colon adenoma based on multivariable logistic regression model. After adjusting for other factors, age, gender, type of attending, and HIV-negative status were significantly associated with having a right colon adenoma. Older patients were significantly more likely to have right colon adenomas than younger patients $(\mathrm{OR}=1.026,95 \%$ CI: $1.01-1.05$, P-value $\left.{ }^{2}=0.0067\right)$.

Table 6 demonstrates the extent to which the ADR of gastroenterologists confounded the other risk factors. It describes the estimated odds ratio and their 95\% confidence intervals of all potential risk factors for each clinical outcome based on three sets of multivariable logistic regression model (1st model: Both "race" and "institution" were used in the model; 2nd model: only "race" was used in the model; 3rd model: only "institution" was used in the model). The significant risk factors from all three multivariable regression models were consistent. For example, based on three sets of multivariable regression models, older people were more likely to have adenoma after controlling other factors (ORs $>1$, P-values $<0.05$ ). Negative HIV status was significantly associated with having an adenoma after controlling for other factors in 1st and 3rd model (P-values $=0.0348,0.031$, respectively). In contrast, HIV was not significantly associated with having an adenoma but the statistical significance was on the border-line ( $\mathrm{P}$-value $=0.0523$ ) based on the 2 nd model.

Table 5. Estimated odds ratio and their $95 \%$ confidence intervals of all potential risk factors for right colon adenoma based on multivariable logistic regression model (c-index: $0.623)$.

\begin{tabular}{|c|c|c|c|}
\hline Variable & Levels & Odds Ratio (95\% CI) & P-value ${ }^{2}$ \\
\hline Age & Every 1 year increase in Age & $1.026(1.01-1.05)$ & 0.0067 \\
\hline BMI & Every 1 unit increase in BMI & $0.986(0.96-1.01)$ & 0.2226 \\
\hline Gender & Female vs Male & $0.694(0.54-0.9)$ & 0.0049 \\
\hline \multirow{6}{*}{ Insurance } & Private vs Medicare & $0.689(0.46-1.04)$ & \multirow{6}{*}{0.0911} \\
\hline & Private vs Medicaid & $1.182(0.84-1.66)$ & \\
\hline & Private vs Self-Pay & $1.154(0.78-1.72)$ & \\
\hline & Medicare vs Medicaid & $1.714(1.12-2.63)$ & \\
\hline & Medicare vs Self-Pay & $1.674(1.03-2.71)$ & \\
\hline & Medicaid vs Self-Pay & $0.977(0.68-1.41)$ & \\
\hline \multirow{3}{*}{ Institution } & DMC vs Kings county & $0.794(0.45-1.41)$ & \multirow{3}{*}{0.1979} \\
\hline & DMC vs SBUMC & $1.482(0.84-2.62)$ & \\
\hline & Kings county vs SBUMC & $1.867(0.93-3.74)$ & \\
\hline \multirow{3}{*}{ Race combined } & White vs Black & $1.337(0.76-2.35)$ & \multirow{3}{*}{0.1355} \\
\hline & White vs Other & $0.751(0.43-1.32)$ & \\
\hline & Black vs Other & $0.561(0.32-0.99)$ & \\
\hline Ethnicity & Hispanic vs Non-Hispanic & $0.978(0.55-1.75)$ & 0.9402 \\
\hline Type of attending & Consultant vs Teaching & $0.577(0.35-0.95)$ & 0.0322 \\
\hline Fellow & Yes vs No & $0.89(0.55-1.44)$ & 0.6373 \\
\hline Tobacco use & Current vs Not current & $1.302(0.86-1.96)$ & 0.2075 \\
\hline Diabetes & Yes vs No & $1.143(0.85-1.53)$ & 0.3706 \\
\hline HIV status & Yes vs No & $0.392(0.15-0.99)$ & 0.0486 \\
\hline
\end{tabular}

${ }^{2} \mathrm{P}$-value was based on type3 analysis from multivariable logistic regression model. 
Table 6. Estimated odds ratio and their $95 \%$ confidence intervals of all potential risk factors for each clinical outcome based on multivariable logistic regression model.

\begin{tabular}{|c|c|c|c|c|c|c|c|c|}
\hline \multirow[b]{2}{*}{ Outcome } & \multirow[b]{2}{*}{ variable } & \multirow[b]{2}{*}{ Levels } & \multicolumn{2}{|c|}{ Both race and institution } & \multicolumn{2}{|c|}{ Only race } & \multicolumn{2}{|c|}{ Only institution } \\
\hline & & & $\begin{array}{c}\text { Odds Ratio } \\
(95 \% \mathrm{CI})\end{array}$ & P-value ${ }^{2}$ & $\begin{array}{l}\text { Odds Ratio } \\
(95 \% \mathrm{CI})\end{array}$ & $P$-value ${ }^{2}$ & $\begin{array}{c}\text { Odds Ratio } \\
(95 \% \mathrm{CI})\end{array}$ & P-value ${ }^{2}$ \\
\hline & Age & Every 1 year increase in Age & $\begin{array}{c}1.027 \\
(1.01-1.04)\end{array}$ & 0.0009 & $\begin{array}{c}1.028 \\
(1.01-1.04)\end{array}$ & 0.0006 & $\begin{array}{c}1.027 \\
(1.01-1.04)\end{array}$ & 0.001 \\
\hline & BMI & Every 1 unit increase in BMI & $\begin{array}{c}0.987 \\
(0.97-1.01)\end{array}$ & 0.1658 & $\begin{array}{c}0.986 \\
(0.97-1)\end{array}$ & 0.1483 & $\begin{array}{c}0.985 \\
(0.97-1)\end{array}$ & 0.1247 \\
\hline & & Private vs Medicare & $\begin{array}{c}0.767 \\
(0.53-1.1)\end{array}$ & & $\begin{array}{c}0.754 \\
(0.53-1.08)\end{array}$ & & $\begin{array}{c}0.778 \\
(0.54-1.12)\end{array}$ & \\
\hline & & Private vs Medicaid & $\begin{array}{c}1.053 \\
(0.79-1.4)\end{array}$ & & $\begin{array}{c}1.04 \\
(0.79-1.38)\end{array}$ & & $\begin{array}{c}1.055 \\
(0.79-1.41)\end{array}$ & \\
\hline & & Private vs Self-Pay & $\begin{array}{c}1.003 \\
(0.71-1.41)\end{array}$ & & $\begin{array}{c}1.047 \\
(0.75-1.46)\end{array}$ & & $\begin{array}{c}1.026 \\
(0.73-1.44)\end{array}$ & \\
\hline & Insurance & Medicare vs Medicaid & $\begin{array}{c}1.373 \\
(0.94-1.99)\end{array}$ & 0.404 & $\begin{array}{c}1.379 \\
(0.95-2)\end{array}$ & 0.3508 & $\begin{array}{c}1.355 \\
(0.93-1.97)\end{array}$ & 0.4401 \\
\hline & & Medicare vs Self-Pay & $\begin{array}{c}1.308 \\
(0.86-2)\end{array}$ & & $\begin{array}{c}1.389 \\
(0.92-2.1)\end{array}$ & & $\begin{array}{c}1.318 \\
(0.86-2.01)\end{array}$ & \\
\hline & & Medicaid vs Self-Pay & $\begin{array}{c}0.953 \\
(0.69-1.31)\end{array}$ & & $\begin{array}{c}1.007 \\
(0.74-1.37)\end{array}$ & & $\begin{array}{c}0.972 \\
(0.71-1.33)\end{array}$ & \\
\hline & & DMC vs Kings county & $\begin{array}{c}1.515 \\
(0.94-2.43)\end{array}$ & & & & $\begin{array}{c}1.521 \\
(0.95-2.43)\end{array}$ & \\
\hline & Institution & DMC vs SBUMC & $\begin{array}{c}1.605 \\
(0.99-2.59)\end{array}$ & 0.0694 & & . & $\begin{array}{c}1.2 \\
(0.87-1.65)\end{array}$ & 0.2029 \\
\hline \multirow[t]{11}{*}{ Adenoma } & & Kings county vs SBUMC & $\begin{array}{c}1.059 \\
(0.59-1.89)\end{array}$ & & & & $\begin{array}{c}0.789 \\
(0.51-1.21)\end{array}$ & \\
\hline & Gender & Female vs Male & $\begin{array}{c}0.626 \\
(0.5-0.78)\end{array}$ & $<0.0001$ & $\begin{array}{c}0.615 \\
(0.5-0.76)\end{array}$ & $<0.0001$ & $\begin{array}{c}0.615 \\
(0.5-0.76)\end{array}$ & $<0.0001$ \\
\hline & & White vs Black & $\begin{array}{c}1.426 \\
(0.89-2.29)\end{array}$ & & $\begin{array}{c}1.094 \\
(0.82-1.47)\end{array}$ & & & \\
\hline & $\begin{array}{c}\text { Race } \\
\text { combined }\end{array}$ & White vs Other & $\begin{array}{c}0.849 \\
(0.53-1.37)\end{array}$ & 0.1116 & $\begin{array}{c}0.781 \\
(0.49-1.24)\end{array}$ & 0.3334 & & \\
\hline & & Black vs Other & $\begin{array}{c}0.596 \\
(0.36-0.98)\end{array}$ & & $\begin{array}{c}0.714 \\
(0.46-1.12)\end{array}$ & & & \\
\hline & Ethnicity & Hispanic vs Non-Hispanic & $\begin{array}{c}0.986 \\
(0.6-1.62)\end{array}$ & 0.957 & $\begin{array}{c}1.062 \\
(0.65-1.74)\end{array}$ & 0.812 & $\begin{array}{c}1.276 \\
(0.84-1.94)\end{array}$ & 0.256 \\
\hline & $\begin{array}{c}\text { Type of } \\
\text { attending }\end{array}$ & Consultant vs Teaching & $\begin{array}{c}0.741 \\
(0.49-1.12)\end{array}$ & 0.1543 & $\begin{array}{c}0.59 \\
(0.45-0.77)\end{array}$ & $<0.0001$ & $\begin{array}{c}0.746 \\
(0.49-1.13)\end{array}$ & 0.1631 \\
\hline & Fellow & Yes vs No & $\begin{array}{c}1.176 \\
(0.8-1.74)\end{array}$ & 0.4155 & $\begin{array}{c}1.001 \\
(0.71-1.41)\end{array}$ & 0.9973 & $\begin{array}{c}1.184 \\
(0.8-1.75)\end{array}$ & 0.3954 \\
\hline & $\begin{array}{c}\text { Tobacco } \\
\text { use }\end{array}$ & Current vs Not current & $\begin{array}{c}1.499 \\
(1.07-2.11)\end{array}$ & 0.0195 & $\begin{array}{c}1.522 \\
(1.09-2.13)\end{array}$ & 0.0147 & $\begin{array}{c}1.485 \\
(1.06-2.08)\end{array}$ & 0.0225 \\
\hline & Diabetes & Yes vs No & $\begin{array}{c}1.265 \\
(0.98-1.63)\end{array}$ & 0.066 & $\begin{array}{c}1.267 \\
(0.99-1.63)\end{array}$ & 0.0644 & $\begin{array}{c}1.254 \\
(0.98-1.61)\end{array}$ & 0.0761 \\
\hline & HIV status & Yes vs No & $\begin{array}{c}0.473 \\
(0.24-0.95)\end{array}$ & 0.0348 & $\begin{array}{c}0.505 \\
(0.25-1.01)\end{array}$ & 0.0523 & $\begin{array}{c}0.466 \\
(0.23-0.93)\end{array}$ & 0.031 \\
\hline
\end{tabular}




\section{Continued}

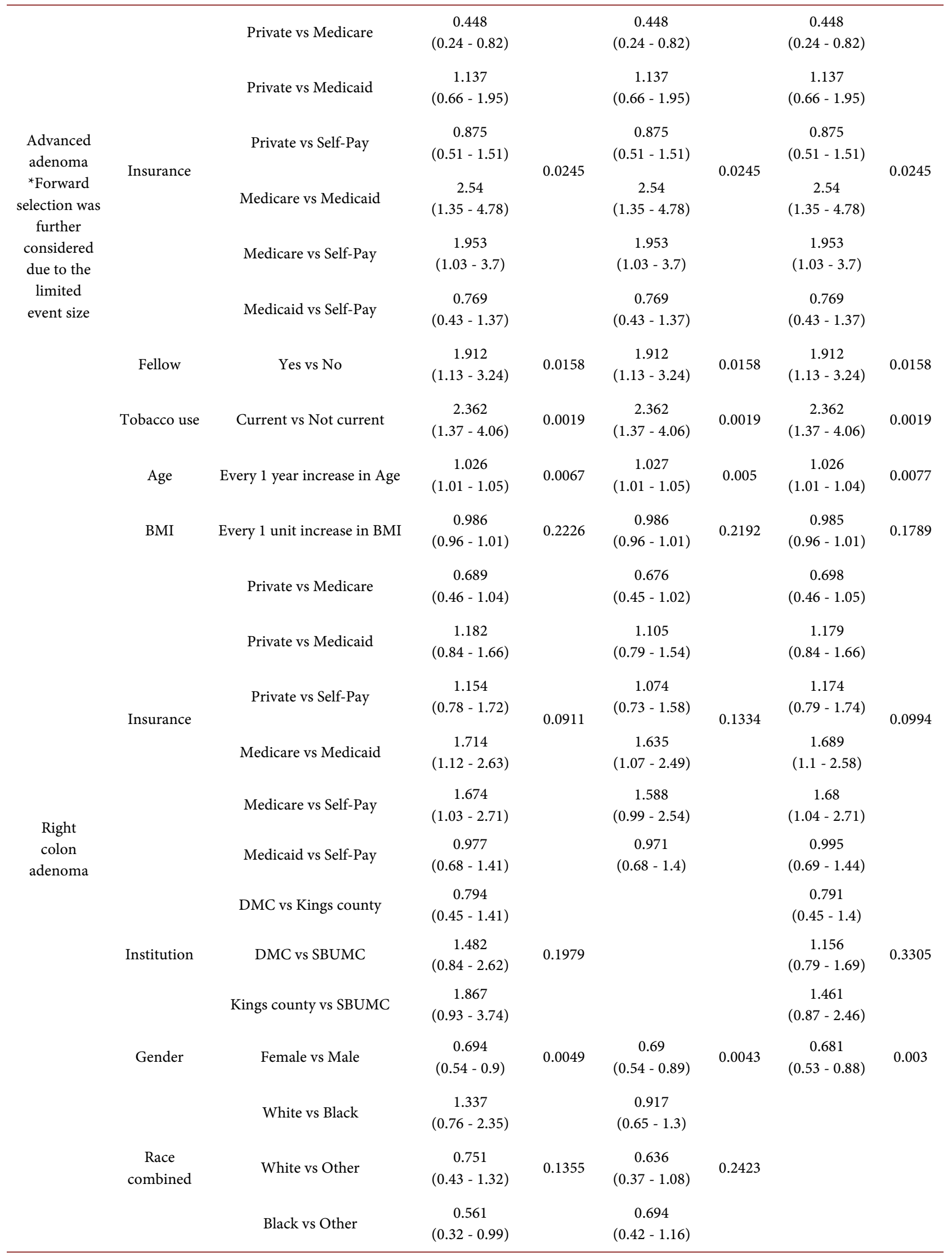




\section{Continued}

\begin{tabular}{|c|c|c|c|c|c|c|c|}
\hline Ethnicity & Hispanic vs Non-Hispanic & $\begin{array}{c}0.978 \\
(0.55-1.75)\end{array}$ & 0.9402 & $\begin{array}{c}1.043 \\
(0.59-1.85)\end{array}$ & 0.8865 & $\begin{array}{c}1.328 \\
(0.82-2.16)\end{array}$ & 0.2545 \\
\hline $\begin{array}{l}\text { Type of } \\
\text { attending }\end{array}$ & Consultant vs Teaching & $\begin{array}{c}0.577 \\
(0.35-0.95)\end{array}$ & 0.0322 & $\begin{array}{c}0.718 \\
(0.53-0.98)\end{array}$ & 0.0354 & $\begin{array}{c}0.58 \\
(0.35-0.96)\end{array}$ & 0.0331 \\
\hline Fellow & Yes vs No & $\begin{array}{c}0.89 \\
(0.55-1.44)\end{array}$ & 0.6373 & $\begin{array}{c}0.996 \\
(0.66-1.5)\end{array}$ & 0.9851 & $\begin{array}{c}0.895 \\
(0.55-1.45)\end{array}$ & 0.6509 \\
\hline $\begin{array}{c}\text { Tobacco } \\
\text { use }\end{array}$ & Current vs Not current & $\begin{array}{c}1.302 \\
(0.86-1.96)\end{array}$ & 0.2075 & $\begin{array}{c}1.266 \\
(0.84-1.9)\end{array}$ & 0.2578 & $\begin{array}{c}1.292 \\
(0.86-1.95)\end{array}$ & 0.2206 \\
\hline Diabetes & Yes vs No & $\begin{array}{c}1.143 \\
(0.85-1.53)\end{array}$ & 0.3706 & $\begin{array}{c}1.155 \\
(0.86-1.55)\end{array}$ & 0.3326 & $\begin{array}{c}1.138 \\
(0.85-1.52)\end{array}$ & 0.3861 \\
\hline HIV status & Yes vs No & $\begin{array}{c}0.392 \\
(0.15-0.99)\end{array}$ & 0.0486 & $\begin{array}{c}0.402 \\
(0.16-1.02)\end{array}$ & 0.0544 & $\begin{array}{c}0.384 \\
(0.15-0.97)\end{array}$ & 0.0437 \\
\hline
\end{tabular}

${ }^{2} \mathrm{P}$-value was based on type3 analysis from multivariable logistic regression model.

\section{Discussion}

In this study our hypothesis that older age, male sex and current smoking were associated with a higher risk of detecting anadenoma was confirmed. These findings concur with those observed in a large study conducted by Kaiser Permanente and a meta-analysis of 18 studies examining risk factors for colon polyps [19] [20].

Given that Black/African-Americans have a higher incidence of colon cancer, our pretest hypothesis was that Black/African-American race would be associated with a higher risk of detecting adenomas. However, in the univariate analysis, Caucasian race was associated with a higher risk and in the multivariable analysis race was not significantly associated with the risk of detecting anadenoma. A similar finding was noted in a smaller study among uninsured patients in New York [21] but is contrary to other larger studies which demonstrated an increased adenoma risk among Black/African-Americans [22] [23] [24] [25]. There are several possible explanations for this finding including differences in sex, smoking status, genetic background and adenoma detection rates of the gastroenterologist.

Blacks/African-Americans receive screening at an older age which would confer a higher risk of adenomas but they had lower rates of the other risk factors such as male sex and current smoking. Additionally, this Black/African-American population may consist of subgroups that inherently have a lower risk for adenomas. Approximately half of the Black/African American patients seen at DMC and $40 \%$ of those at Kings County were documented as Afro Caribbean [26]. The risk of colon malignancy and polyps in Afro-Caribbean subjects may be different from Black/African-Americans born in the US and may be similar to Non-Hispanic Caucasians as reported in a Florida based study [27]. We were unable to perform subgroup analysis on the Black/African American population in our study as country of origin was not consistently documented at all of the sites. 
Another factor that likely contributed to the lack of association between race and adenomas is that the analysis may have been confounded by significant differences in the ADR of the gastroenterologists performing the procedures. A significantly higher proportion of colonoscopies among Black/African-American patients was performed by non-teaching or consultant gastroenterologists who had a significantly lower ADR at one of the three institutions. A recent study on interval colon cancer in Medicare enrollees noted that a higher proportion of black persons (52.8\%) than white persons (46.2\%) received colonoscopies from physicians with a lower Polyp Detection Rate [28]. This rate was significantly associated with interval CRC risk.

A recent joint task force of the American College of Gastroenterology and the American Society of Gastrointestinal Endoscopy recommended ADR benchmarks of $25 \%$ for all patients and sex-specific rates of $30 \%$ for men and $20 \%$ for women [29]. Our study included colonoscopies performed during 2012, when the benchmark adenoma detection rate was $20 \%$ overall (15\% female and $25 \%$ male). Our finding of a significant difference in ADR between teaching and contracted consultant gastroenterologists reinforces the concept that detection of colon polyps is operator dependent [30] [31] as we controlled for the patient factors that contribute to lower ADR such as bowel prep quality and cecal intubation rate. Unfortunately cecal withdrawal time was not recorded consistently so we were unable to control for this.

Various colonoscopy screening programs have been implemented to improve access of uninsured and minority patients to screening colonoscopies [32] [33]. While rates of colonoscopy completion have been used as measures of success of these programs, this finding in our study indicates the importance of continued surveillance of the quality of these colonoscopies to ensure that the optimal benefit is being achieved. Implementation of quality metric monitoring and direct feedback to gastroenterologists has been shown to improve ADR [34] [35] and this has been implemented at all the institutions in this study.

The lack of any association with Black/African-American race and proximal adenoma is contrary to the observed distribution of right sided colon cancers in this population [20] [36] [37] [38] [39] [40] and concurs with the findings of a similar study conducted by Freidburg et al. [41]. Either sample size or operator dependence could also have affected our results regarding the detection of proximal adenomas.

There have been conflicting observations of the prevalence of advanced adenomas in Black/African-American patients [39] [40] [41] and no association was observed in our study. The effect of operator dependence may have had less of an effect for this metric since most of the advanced adenomas were larger in size. One study has suggested that advanced adenoma detection is independent of ADR [42].

The American Cancer Society updated their CRC screening recommendations in May 2018 to initiate screening for all patients at age 45 years [43]. However this recommendation has been in place for African-Americans since 2009 by 
multiple societies [44]. However in our study the median age of initial screening among Black/African American patients was significantly higher than Caucasian patients. One reason for this is that acceptance of screening colonoscopies may be lower than the alternative of annual fecal immunochemical testing (FIT) in the Black/African/American population [45]. Furthermore it should be noted that current programs supporting free colorectal screening (largely FIT based), do not support initiating screening for individuals under the age of 50 [46].

Although it is hypothesized that HIV infection increases the risk of Non-AIDS defining malignancies [47], a meta-analysis of previous studies shows no association between HIV infection and colorectal cancer [48]. Conflicting results have been reported regarding the relationship between HIV infection and the detection of adenoma [49] [50] [51]. In our study, no association between adenomas was observed in HIV-infected individuals. Discrepant findings may be as a result of the small number of HIV-infected patients in these studies, and may also be as a result of lower CRC screening rates among HIV-infected patients [52].

One of the major strengths of this study is the sample size and the representation of Black/African-Americans in the sample which allowed for comparisons of multiple variables with the Caucasian population. Additionally, the exclusion criteria ensured that only patients with average risk screening colonoscopies were included. The exclusion criteria also removed other determinants of ADR as incomplete studies and those with inadequate prep.

A major limitation of our study is the variation in ADR due to the type of gastroenterologist during 2012 which would have impacted the effect of other variables on detection of adenomas. Additionally, there are recent observations that some proximal serrated adenomas may have been misclassified as hyperplastic polyps and this is variable amongst pathologists [53] [54] [55]. Due to the retrospective design of the study we were unable to control for this variability in pathologists. In our analysis we excluded all hyperplastic polyps regardless of site which may have resulted in an underestimation of adenoma.

\section{Conclusion}

In this study male sex, older age, current smoking and diabetes were associated with increased prevalence of adenoma. This finding may have been influenced by disparities in the ADR of gastroenterologists performing screening in the Black/African American populations. However, initiatives to improve quality have been implemented across all the institutions. Now that the effect of operator dependence has been greatly reduced, we plan to resume collection of data for this study beginning with 2019 to better define the populations at higher risk of adenoma. Further studies delineating the biologic factors including the microbiome affecting adenomas should also be conducted. The hope is that early preventive interventions to reduce the prevalence of these risk factors and treatment options targeting them may further reduce colon cancer incidence and mortality in this population. 


\section{Acknowledgements}

We wish to thank all of the patients who contributed to this study and will continue to contribute to further studies. We thank Dr Moro Salifu for facilitating the collaboration between Stony Brook University and SUNY Downstate Medical Center. We also thank Jennifer Caceres, MD, Karthik Raghunathan, MD, Kirolos Iskander, MD, Michael Mann, Khalid Awwal for their assistance with data collection. We also acknowledge the biostatistical consultation and biostatistical support provided by the Biostatistical Consulting Core at School of Medicine, Stony Brook University. We would also like to thank those at NYC HHC Corp who reviewed and made constructive comments: Machelle Allen, MD, Steven Pulitzer, MD and Wendy Wilcox, MD.

\section{Specific Author Contributions}

Study concept and design: Joshua Miller, Ellen Li, Yakira David, Lorenzo Ottaviano. Data collection: Yakira David, Ellen Li, Lorenzo Ottaviano, Michelle Likhtshteyn, Sadat Iqbal, Samir Kumar, Brandon Lung, Helen Lyo, Jesse T. Frye, Ayanna E. Lewis. Analysis and Interpretation of Data: Ellen Li, Jihye Park, Jie Yang, Yakira David, Joshua Miller, Lorenzo Ottaviano,. Drafting of the Manuscript: Ellen Li, Yakira David, Michele Follen, Evan Grossman. Critical Review for Important Intellectual Content: Joshua Miller, Lorenzo Ottaviano, ShivakuarVignesh, Evan Grossman, Laura Martello, Ayanna E. Lewis, Michele Follen. Study Supervision: Laura Martello, Joshua Miller, Ellen Li, ShivakumarVignesh, Evan Grossman.

\section{Financial Support}

NCI P20 CA192994 (E.L.), Simons Foundation (E.L.), Stony Brook FUSION Seed Grant Award (J.D.M.). The work conducted is independent of the funding source.

\section{Guarantor of Article}

Evan Grossman MD.

\section{Conflicts of Interest}

The authors declare no conflict of interest.

\section{References}

[1] Howlander, N.N.A., Krapcho, M., Miller, D., Bishop, K., Kosary, C.L., Yu, M., Ruhl, J., Tatalovich, Z., Mariotto, A., Lewis, D.R., Chen, H.S., Feuer, E.J. and Cronin, K.A. (2016) SEER Cancer Statistics Review, 1975-2014. Institute NC, Bethesda, MD.

[2] Haggar, F.A. and Boushey, R.P. (2009) Colorectal Cancer Epidemiology: Incidence, Mortality, Survival, and Risk Factors. Clinics in Colon and Rectal Surgery, 22, 191-197. https://doi.org/10.1055/s-0029-1242458

[3] Colussi, D., Brandi, G., Bazzoli, F. and Ricciardiello, L. (2013) Molecular Pathways 
Involved in Colorectal Cancer: Implications for Disease Behavior and Prevention. International Journal of Molecular Sciences, 14, 16365-16385. https://doi.org/10.3390/ijms140816365

[4] Pino, M.S. and Chung, D.C. (2010) The Chromosomal Instability Pathway in Colon Cancer. Gastroenterology, 138, 2059-2072.

https://doi.org/10.1053/j.gastro.2009.12.065

[5] Zauber, A.G. (2015) The Impact of Screening on Colorectal Cancer Mortality and Incidence-Has It Really Made a Difference? Digestive Diseases and Sciences, 60, 681-691.

[6] Lin, O.S., Kozarek, R.A. and Cha, J.M. (2014) Impact of Sigmoidoscopy and Colonoscopy on Colorectal Cancer Incidence and Mortality: An Evidence-Based Review of Published Prospective and Retrospective Studies. Intestinal Research, 12, 268-274. https://doi.org/10.5217/ir.2014.12.4.268

[7] Shaukat, A. and Church, T.R. (2013) Colorectal-Cancer Incidence and Mortality after Screening. The New England Journal of Medicine, 369, 2355.

https://doi.org/10.1056/NEJMoa1300720

[8] Shaukat, A., Mongin, S.J., Geisser, M.S., et al. (2013) Long-Term Mortality after Screening for Colorectal Cancer. The New England Journal of Medicine, 369, 1106-1114. https://doi.org/10.1056/NEJMoa1300720

[9] Johnson, C.M., Wei, C., Ensor, J.E., et al. (2013) Meta-Analyses of Colorectal Cancer Risk Factors. Cancer Causes and Control, 24, 1207-1222. https://doi.org/10.1007/s10552-013-0201-5

[10] Giovannucci, E. and Willett, W.C. (1994) Dietary Factors and Risk of Colon Cancer. Annals of Medicine, 26, 443-452. https://doi.org/10.3109/07853899409148367

[11] Ullman, T.A. and Itzkowitz, S.H. (2011) Intestinal Inflammation and Cancer. Gastroenterology, 140, 1807-1816. https://doi.org/10.1053/j.gastro.2011.01.057

[12] Siegel, R., Naishadham, D. and Jemal, A. (2012) Cancer Statistics, 2012. CA: $A$ Cancer Journal for Clinicians, 62, 10-29. https://doi.org/10.3322/caac.20138

[13] O’Keefe, S.J.D., Li, J.V., Lahti, L., et al. (2015) Fat, Fibre and Cancer Risk in African Americans and Rural Africans. Nature Communications, 6, 6342. https://doi.org/10.1038/ncomms7342

[14] O'Keefe, S.J., Chung, D., Mahmoud, N., et al. (2007) Why Do African Americans Get More Colon Cancer than Native Africans? The Journal of Nutrition, 137, 175S-182S.

[15] Brim, H. and Ashktorab, H. (2016) Genomics of Colorectal Cancer in African Americans. Journal of Next Generation Sequencing and Applications, 3, 133. https://doi.org/10.4172/2469-9853.1000133

[16] Li, E., Ji, P., Ouyang, N., et al. (2014) Differential Expression of miRNAs in Colon Cancer between African and Caucasian Americans: Implications for Cancer Racial Health Disparities. International Journal of Oncology, 45, 587-594. https://doi.org/10.3892/ijo.2014.2469

[17] Dimou, A., Syrigos, K.N. and Saif, M.W. (2009) Disparities in Colorectal Cancer in African-Americans vs Whites: Before and after Diagnosis. World Journal of Gastroenterology 15, 3734-3743. https://doi.org/10.3748/wjg.15.3734

[18] Ollberding, N.J., Nomura, A.M., Wilkens, L.R., Henderson, B.E. and Kolonel, L.N. (2011) Racial/Ethnic Differences in Colorectal Cancer Risk: The Multiethnic Cohort Study. International Journal of Cancer, 129, 1899-1906.

https://doi.org/10.1002/ijc.25822 
[19] Heitman, S.J., Ronksley, P.E., Hilsden, R.J., et al. (2009) Prevalence of Adenomas and Colorectal Cancer in Average Risk Individuals: A Systematic Review and Meta-Analysis. Clinical Gastroenterology and Hepatology, 7, 1272-1278. https://doi.org/10.1016/j.cgh.2009.05.032

[20] Corley, D.A., Jensen, C.D., Marks, A.R., et al. (2013) Variation of Adenoma Prevalence by Age, Sex, Race, and Colon Location in a Large Population: Implications for Screening and Quality Programs. Clinical Gastroenterology and Hepatology, 11, 172-180. https://doi.org/10.1016/j.cgh.2012.09.010

[21] Collazo, T.H., Jandorf, L., Thelemaque, L., et al. (2015) Screening Colonoscopy among Uninsured and Underinsured Urban Minorities. Gut and Liver, 9, 502-508. https://doi.org/10.5009/gnl14039

[22] Nouraie, M., Hosseinkhah, F., Zamanifekri, B., et al. (2010) Clinicopathological Features Colon Polyps from African-Americans. Digestive Diseases and Sciences, 55, 1442-1449. https://doi.org/10.1007/s10620-010-1133-5

[23] Thornton, J.G., Morris, A.M., Thornton, J.D., et al. (2007) Racial Variation in Colorectal Polyp and Tumor Location. Journal of the National Medical Association, 99, 723-728.

[24] Rex, D.K., Khan, A.M., Shah, P., Newton, J. and Cummings, O.W. (2000) Screening Colonoscopy in Asymptomatic Average-Risk African Americans. Gastrointestinal Endoscopy, 51, 524-527. https://doi.org/10.1016/S0016-5107(00)70283-5

[25] Lebwohl, B., Capiak, K., Neugut, A.I. and Kastrinos, F. (2012) Risk of Colorectal Adenomas and Advanced Neoplasia in Hispanic, Black and White Patients Undergoing Screening Colonoscopy. Alimentary Pharmacology and Therapeutics, 35, 1467-1473. https://doi.org/10.1111/j.1365-2036.2012.05119.x

[26] Kings County Hospital (2013) Community Health Needs Assessment and Implementation Strategy.

[27] Alvarez, A., Tandon, K., Castro, F.J.S., et al. (2016) Comparison of Adenoma Detection Rates in Afro-Caribbeans and Non-Hispanic Whites Undergoing First Screening Colonoscopy. The Southern Medical Journal, 109, 277-281. https://doi.org/10.14423/SMJ.0000000000000456

[28] Fedewa, S.A., Flanders, W.D., Jemal, A., et al. (2018) Racial and Ethnic Disparities in Interval Colorectal Cancer Incidence: A Population-Based Cohort Study. Annals of Internal Medicine, 166, 857-866. https://doi.org/10.7326/M16-1154

[29] Rex, D.K., Bond, J.H., Winawer, S., et al. (2002) Quality in the Technical Performance of Colonoscopy and the Continuous Quality Improvement Process for Colonoscopy: Recommendations of the U.S. Multi-Society Task Force on Colorectal Cancer. The American Journal of Gastroenterology, 97, 1296-1308.

[30] Adler, A., Wegscheider, K., Lieberman, D., et al. (2013) Factors Determining the Quality of Screening Colonoscopy: A Prospective Study on Adenoma Detection Rates, from 12,134 Examinations (Berlin Colonoscopy Project 3, BECOP-3). Gut, 62, 236-241. https://doi.org/10.1136/gutjnl-2011-300167

[31] Mohammed, N. and Subramanian, V. (2013) Screening Colonoscopy: Should We Focus More on Technique and Less on Technology? F1000 Prime Reports, 5, 32.

[32] Centers for Disease Control and Prevention (2015) Cancer Prevention Works: Spotlight on Year 1 of CDC's Colorectal Cancer Control Program.

[33] Itzkowitz, S.H., Winawer, S.J., Krauskopf, M., et al. (2016) New York Citywide Colon Cancer Control Coalition: A Public Health Effort to Increase Colon Cancer Screening and Address Health Disparities. Cancer, 122, 269-277. 
https://doi.org/10.1002/cncr.29595

[34] Gurudu, S.R., Boroff, E.S., Crowell, M.D., et al. (2018) Impact of Feedback on Adenoma Detection Rates: Outcomes of Quality Improvement Program. Journal of Gastroenterology and Hepatology, 33, 645-649. https://doi.org/10.1111/jgh.13984

[35] Kahi, C.J., Ballard, D., Shah, A.S., et al. (2013) Impact of a Quarterly Report Card on Colonoscopy Quality Measures. Gastrointestinal Endoscopy, 77, 925-931. https://doi.org/10.1016/j.gie.2013.01.012

[36] Mostafa, G., Matthews, B.D., Norton, H.J., Kercher, K.W., Sing, R.F. and Heniford, B.T. (2004) Influence of Demographics on Colorectal Cancer. The American Journal of Surgery, 70, 259-264.

[37] Chattar-Cora, D., Onime, G.D., Valentine, I.S., Cudjoe, E. and Rivera, L. (2000) Colorectal Cancer in a Multi-Ethnic Urban Group: Its Anatomical and Age Profile. International Journal of Surgery, 85, 137-142.

[38] Jackson, C.S. and Vega, K.J. (2015) Higher Prevalence of Proximal Colon Polyps and Villous Histology in African-Americans Undergoing Colonoscopy at a Single Equal Access Center. Journal of Gastrointestinal Oncology, 6, 638-643.

[39] Lieberman, D.A., Holub, J.L., Moravec, M.D., et al. (2008) Prevalence of Colon Polyps Detected by Colonoscopy Screening in Asymptomatic Black and White Patients. The Journal of the American Medical Association, 300, 1417-1422. https://doi.org/10.1001/jama.300.12.1417

[40] Lieberman, D.A., Williams, J.L., Holub, J.L., et al. (2014) Race, Ethnicity, and Sex Affect Risk for Polyps $>9 \mathrm{~mm}$ in Average-Risk Individuals. Gastroenterology, 147, 351-358. https://doi.org/10.1053/j.gastro.2014.04.037

[41] Friedenberg, F.K., Singh, M., George, N.S., Sankineni, A. and Shah, S. (2012) Prevalence and Distribution of Adenomas in Black Americans Undergoing Colorectal Cancer Screening. Digestive Diseases and Sciences, 57, 489-495. https://doi.org/10.1007/s10620-011-1952-Z

[42] Greenspan, M., Rajan, K.B., Baig, A., et al. (2013) Advanced Adenoma Detection Rate Is Independent of Nonadvanced Adenoma Detection Rate. The American Journal of Gastroenterology, 108, 1286-1292.

[43] Wolf, A.M.D., Fontham, E.T.H., Church, T.R., et al. (2018) Colorectal Cancer Screening for Average-Risk Adults: 2018 Guideline Update from the American Cancer Society. CA: A Cancer Journal for Clinicians, 68, 250-281. https://doi.org/10.3322/caac.21457

[44] Rex, D.K., Johnson, D.A., Anderson, J.C., et al. (2009) American College of Gastroenterology Guidelines for Colorectal Cancer Screening 2009 [Corrected]. The American Journal of Gastroenterology, 104, 739-750.

[45] DeBourcy, A.C., Lichtenberger, S., Felton, S., et al. (2008) Community-Based Preferences for Stool Cards versus Colonoscopy in Colorectal Cancer Screening. Journal of General Internal Medicine, 23, 169-174. https://doi.org/10.1007/s11606-007-0480-1

[46] New York State Cancer Services Program (2016) New York State Department of Health: Bureau of Cancer Prevention and Control.

[47] Silverberg, M.J., Chao, C., Leyden, W.A., et al. (2009) HIV Infection and the Risk of Cancers with and without a Known Infectious Cause. AIDS, 23, 2337-2345. https://doi.org/10.1097/QAD.0b013e3283319184

[48] O’Neill, T.J., Nguemo, J.D., Tynan, A.M., Burchell, A.N. and Antoniou, T. (2017) Risk of Colorectal Cancer and Associated Mortality in HIV: A Systematic Review 
and Meta-Analysis. Journal of Acquired Immune Deficiency Syndromes, 75, 439-447. https://doi.org/10.1097/QAI.0000000000001433

[49] Bini, E.J., Park, J. and Francois, F. (2006) Use of Flexible Sigmoidoscopy to Screen for Colorectal Cancer in HIV-Infected Patients 50 Years of Age and Older. Archives of Internal Medicine, 166, 1626-1631. https://doi.org/10.1001/archinte.166.15.1626

[50] Gutkin, E., Hussain, S.A., Mehta, P., et al. (2012) Prevalence of Adenomas Found on Colonoscopy in Patients with HIV. Gastroenterology Research, 5, 52-56. https://doi.org/10.4021/gr433w

[51] Fantry, L.E., Nowak, R.G., Fisher, L.H., et al. (2016) Colonoscopy Findings in HIV-Infected Men and Women from an Urban U.S. Cohort Compared with Non-HIV-Infected Men and Women. AIDS Research and Human Retroviruses, 32, 860-867. https://doi.org/10.1089/aid.2015.0322

[52] Reinhold, J.P., Moon, M., Tenner, C.T., Poles, M.A. and Bini, E.J. (2005) Colorectal Cancer Screening in HIV-Infected Patients 50 Years of Age and Older: Missed Opportunities for Prevention. American Journal of Gastroenterology, 100, 1805-1812.

[53] Singh, H., Bay, D., Bernstein, C.N., et al. (2012) Pathological Reassessment of Hyperplastic Colon polyps in a City-Wide Pathology Practice: Implications for Polyp Surveillance Recommendations. Gastrointestinal Endoscopy, 76, 1003-1008. https://doi.org/10.1016/j.gie.2012.07.026

[54] Schoen, R.E. and Akpan, I.M. (2016) Whither the Hyperplastic and Serrated Polyp? Gastrointestinal Endoscopy, 83, 563-565.

[55] Gourevitch, R.A., Rose, S., Crockett, S.D., et al. (2018) Variation in Pathologist Classification of Colorectal Adenomas and Serrated Polyps. American Journal of Gastroenterology, 113, 431-439. 